\title{
Screw fixation in stemless shoulder arthroplasty for the treatment of primary osteoarthritis leads to less osteolysis when compared to impaction fixation
}

\author{
Arad Alikhah', Jan-Phillipp Imiolczyk ${ }^{1}$, Anna Krukenberg ${ }^{1}$ and Markus Scheibel ${ }^{1} 2^{*}$
}

\begin{abstract}
Background: Stemless total shoulder arthroplasty is a well-established and reliable surgical treatment option for glenohumeral osteoarthritis resulting in loss of pain and improvement of shoulder function. Currently the two methods for the fixation of the humeral component are either screw fixation or impaction. The purpose of this study is the clinical and radiological comparison of two different stemless designs (screw fixation vs impaction) for total shoulder arthroplasties in patients suffering from primary glenohumeral osteoarthritis.

Methods: A retrospective cohort study including 39 patients with a mean age of 67 years and a minimum followup of 2 years was performed. Patients were separated into two groups based on the selected implant. In group A $(n=18)$ a screw fixation design and in group $\mathrm{B}(n=21)$ an impaction type design was used. For clinical examination the Constant-Murley-Score (CS) and Subjective-Shoulder-Value (SSV) were evaluated. Radiological examination was performed on true-AP, axial and Y-view radiographs.

Results: In group A the CS increased from 27.1 to 65.2 points and SSV from 27.3 to $76.7 \%(p<0.05)$. No osteolysis of the medial calcar or subsidence of the humeral implant were found in this group. In group B the CS increased from 29.0 to 72.6 points and SSV from 33.1 to $85 \%(p<0.05)$. Osteolysis of the medial calcar was present in seven patients in this group. No signs for humeral loosening were found in both groups.
\end{abstract}

Conclusion: Impaction and screw fixation total shoulder arthroplasty for primary glenohumeral osteoarthritis using a stemless device provide reliable clinical results. The screw fixation seems to prevent osteolysis of the medial calcar.

Keywords: Stemless shoulder arthroplasty, Primary osteoarthritis, Screw fixation, Impaction design

\footnotetext{
* Correspondence: markus.scheibel@charite.de

${ }^{1}$ Department of Shoulder and Elbow Surgery, Center for Musculoskeletal Surgery, Charité-Universitaetsmedizin Berlin, Augustenburger Platz 1, 13353 Berlin, Germany

${ }^{2}$ Department of Shoulder and Elbow Surgery, Schulthess Clinic, Zuerich,

Switzerland
}

c) The Author(s). 2020, corrected publication 2020. Open Access This article is licensed under a Creative Commons Attribution 4.0 International License, which permits use, sharing, adaptation, distribution and reproduction in any medium or format, as long as you give appropriate credit to the original author(s) and the source, provide a link to the Creative Commons licence, and indicate if changes were made. The images or other third party material in this article are included in the article's Creative Commons licence, unless indicated otherwise in a credit line to the material. If material is not included in the article's Creative Commons licence and your intended use is not permitted by statutory regulation or exceeds the permitted use, you will need to obtain permission directly from the copyright holder. To view a copy of this licence, visit http://creativecommons.org/ licenses/by/4.0/. The Creative Commons Public Domain Dedication waiver (http://creativecommons.org/publicdomain/zero/1. 0/) applies to the data made available in this article, unless otherwise stated in a credit line to the data. 


\section{Background}

Total shoulder arthroplasty is a well-established and reliable surgical treatment option for glenohumeral osteoarthritis resulting in loss of pain and improvement of shoulder function [1]. Due to excellent clinical results usage and therefore also revisions become more frequent [2-4]. Stemless shoulder replacement was introduced to shorten operation time, reduce stemmed related complications, save bone stock and ultimately make revisions easier [5-7]. Stemless endoprosthesis are implanted through humeral anchoring in the epiphyseal and/or metaphyseal bone and achieve a canal sparing fixation $[8,9]$. A number of studies have shown radiological reliability and clinical improvements that are comparable with stemmed designs in short and midterm follow-up $[6,10-12]$. Currently there are two methods of humeral fixation. Designs use either an impaction method for anchoring the humeral component or a hollow screw [9, $13,14]$.

The purpose of this study is the clinical and radiological comparison of two different stemless design (impaction vs. screw fixation) of stemless total shoulder arthroplasties for patients with primary glenohumeral osteoarthritis. Our null hypothesis was that there is no difference in clinical and radiological results between the two implants.

\section{Methods}

A retrospective cohort comparison including patients with primary osteoarthritis of the glenohumeral joint was used for this study. This study was approved by the ethics committee of our institution (EA2/154/18). Written informed consent was obtained from all patients enrolled in this study. Inclusion and exclusion criteria are listed in Table 1. Of the 39 included patients 11 were male and 28 were female. Mean age was 67 (44-83 median: 69) years. All patients were evaluated clinically and radiologically with a standardised protocol with a minimum of 2 years follow-up. Clinical outcomes were documented using the Constant-and-Murley Score (CS) [15]. The overall satisfaction was evaluated using the
Subjective-Shoulder-Value (SSV). Standard radiographs (true anterior-posterior, axial and Y-views) were taken to evaluate for signs of loosening or osteolysis according to previous studies $[6,16,17]$. For the evaluation of glenoid loosening the Molé-Score was used [16]. For the radiological evaluation we always compared the 6 weeks postoperative and the last follow-up radiographs. Additionally, intra- and postoperative complications were documented. Patients received the modell of endoprothesis based on which year they were treated. Initially we used a screw fixation method in our clinic, later we switched to an impaction method.

\section{Surgical procedure}

All surgeries were performed by the same senior surgeon. Patients underwent general anaesthesia combined with an interscalene block for optimal pain relief. All patients were placed in beach-chair position. A deltopectoral approach and a subscapularis tenotomy was used in all procedures. A capsular release and a tenotomy of the long head of the biceps tendon was performed and the humeral osteophytes were removed. Implantation of the endoprosthesis was performed following the manufactures description. Two different models of stemless shoulder arthroplasties were implanted.

In the study group (group A) $(n=18)$ the Eclipse Shoulder Prosthesis (Arthrex, Inc., Naples, FL, USA) was used (Fig. 1a-h). This system consists of three humeral components. A central hollow screw sometimes referred to as a cage that fixates a baseplate also called trunion to the anatomical neck. The third component is the humeral head. There are different resection guides corresponding to different sizes of humeral heads. The retroversion was determined according to the patient's anatomic neck and the guide attached with a Steinmann pin leading the way for two K-wires in the proximal humerus. Once the $\mathrm{K}$-wires were placed the resection guide was removed and the humeral head resected at the anatomic neck. Using templates, the trunion size was determined. After preparation of hole for the cage screw using a reamer a protection plate was placed on

Table 1 Patients inclusion and exclusion criteria for this study

\begin{tabular}{ll}
\hline Inclusion criteria & Exclusion criteria \\
\hline Patients are at least 18 years old / Skelettaly mature & $\begin{array}{l}\text { Any other shoulder related pathologies besides primary } \\
\text { osteoarthritis of the glenohumeral joint }\end{array}$ \\
$\begin{array}{l}\text { The patient is willing and able to cooperate with the required } \\
\text { postoperative therapy }\end{array}$ & Disagrees with participation in the study \\
Was diagnosed with primary osteoarthritis of the glenohumeral & \\
joint and required total shoulder arthroplasty & \\
Complete clinical and radiographic examinations at each required & \\
appointment & \\
Minimal Follow-up of 24 months &
\end{tabular}



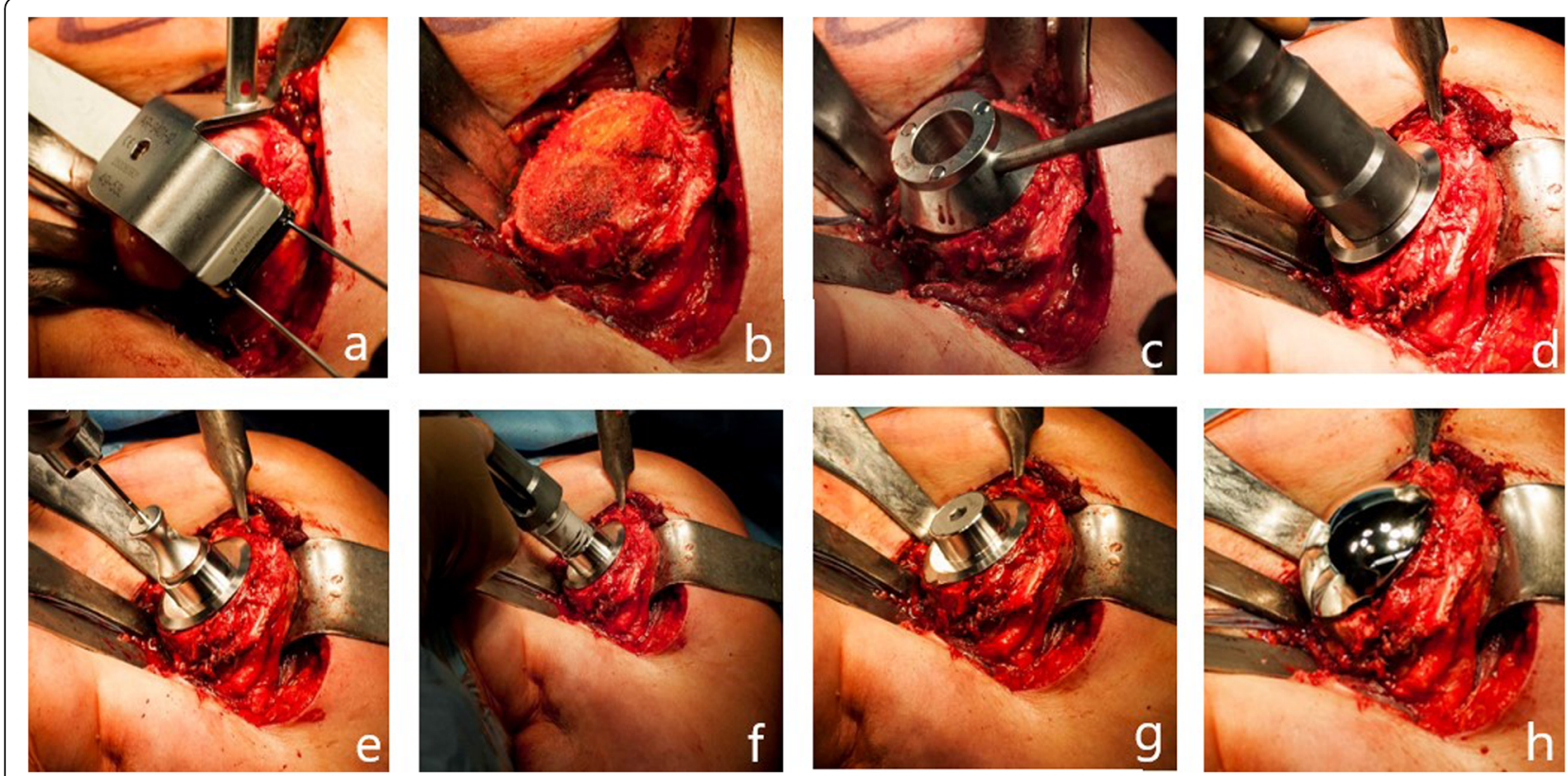

Fig. 1 a-h Surgical technique of the Eclipse Shoulder Prosthesis

the resection area during replacement of the glenoid. Afterwards a cage screw sizer was drilled through a centering device until it reaches the lateral cortex. This process determined the length of the screw. Drill template and cage screw sizer were removed and the baseplate was fixed over the centering devise using an impactor. The cage screw is screwed in over the trunion while pressing the trunion to the resection area. The appropriate head was determined with the help of trial heads. Finally the humeral head is impacted on the trunion.

In the control group (group B) $(n=21)$ the Sidus Stem-free Shoulder System (Zimmer Biomet, Inc., Warsaw, Indiana, USA) was used (Fig. 2a-h). This system is comprised of two parts. A grit blasted titanium anchor and a cobalt chrome humeral head. For resection of the humeral head a guide was used. This guide was
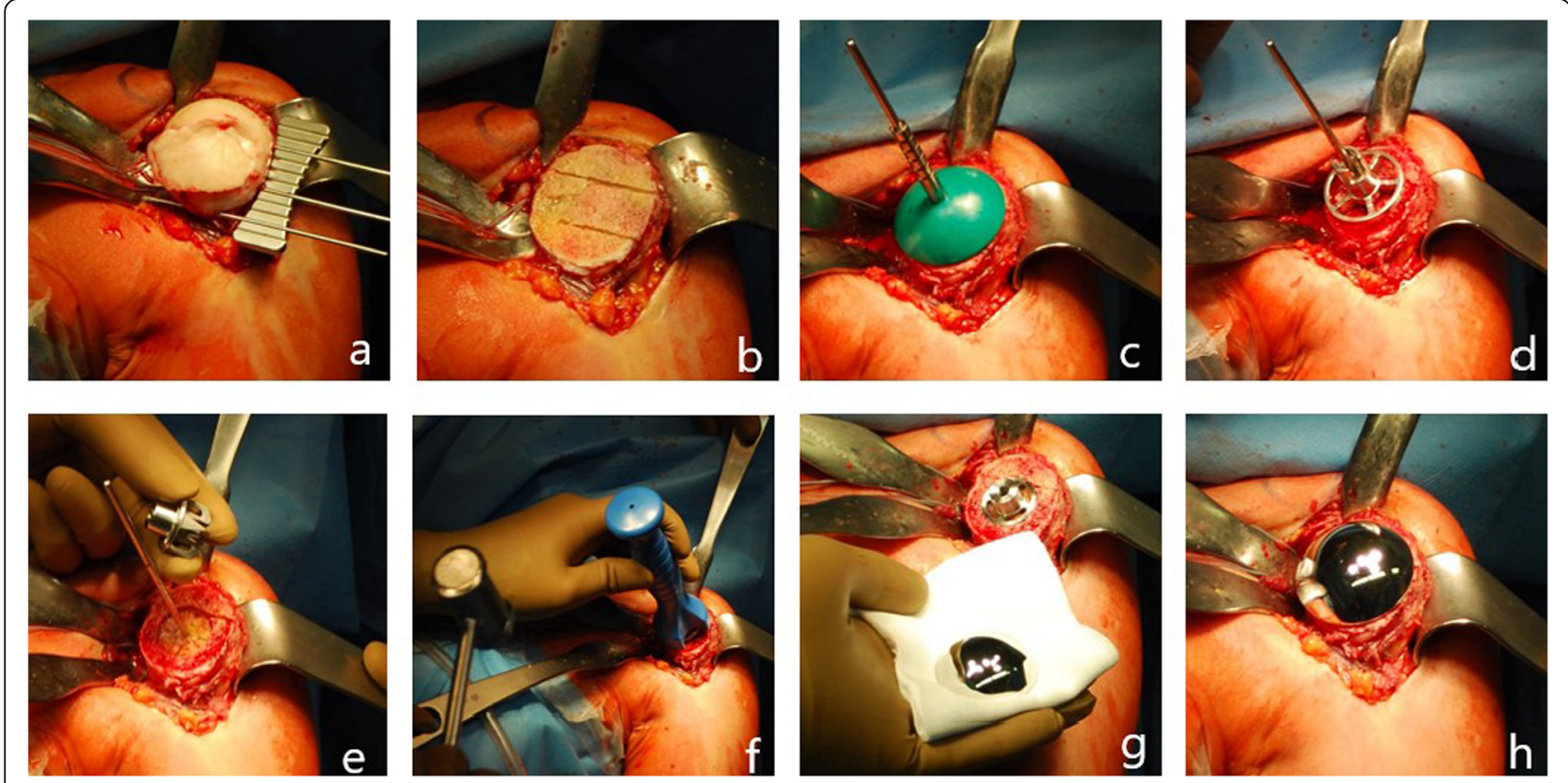

Fig. 2 a-h Surgical technique of Sidus Stem-Free Shoulder System 
Table 2 Compared demographic data between both groups

\begin{tabular}{llll}
\hline & Group A & Group B & $p$ value \\
\hline$N$ & 18 & 21 & \\
Mean age in years & $66.7(44-81)$ & $67.4(55-83)$ & 0.835 \\
Mean follow-up in months & 42.2 & 36.3 & 0.010 \\
\hline
\end{tabular}

positioned at the medial border of the insertion of the supraspinatus tendon using two K-wires. The inclination angle and retroversion were determined with the help of this resection guide. After resection at the anatomic neck a protection plate was placed on the resection plane during preparation of the glenoid. Once the glenoid was replaced a central pin was placed through a trial head. This pin was used for preparation of the humeral fixation side. After a slightly undersized preparation of the humeral bone with a drill and a puncher the anchor was impacted into the humeral head through the same pin. Three different sizes of anchors are available. After testing with a trial head, the final cobalt chrome head is placed on the anchor. The anchor and head are connected by a Morse taper connection.

Both systems were combined with all polyethylene fully cemented keel glenoid. The labrum was resected and the capsule was released around the glenoid and the glenoid was prepared using a reamer and a keel punch. The size of the glenoid was determined with the help of trial glenoids. The definite glenoid was implanted with a high-pressure cement application into the bone and application on the backside of the polyethylene keel glenoid including the keel. The glenoid component was then impacted into the bone. After all arthroplasties were implanted subscapularis repair and wound closure were performed in a standard fashion.

\section{Postoperative rehabilitation}

Postoperatively patients followed a standardized rehabilitation protocol. The shoulder was immobilised in a sling in internal rotation for 6 weeks. During this time, only passive movement (excluding external rotation) above zero degrees was allowed during physiotherapy sessions. Movement was slowly increased under supervision of

Table 3 Preoperative and postoperative clinical results of both groups

\begin{tabular}{llll}
\hline & Group A & Group B & p value \\
\hline $\begin{array}{l}\text { Constant- Murley-Score } \\
\text { (preoperative) }\end{array}$ & 27.1 points & 29 points & 0.762 \\
$\begin{array}{l}\text { Constant-Murley-Score } \\
\text { (last Follow-up) }\end{array}$ & 65.2 points & 72.6 points & 0.167 \\
$\begin{array}{l}\text { Subjective-Shoulder-Value } \\
\text { (preoperative) }\end{array}$ & $27.3 \%$ & $33.1 \%$ & 0.432 \\
$\begin{array}{l}\text { Subjective-Shoulder-Value } \\
\text { (last Follow-up) }\end{array}$ & $76.7 \%$ & $85 \%$ & 0.378 \\
\hline
\end{tabular}

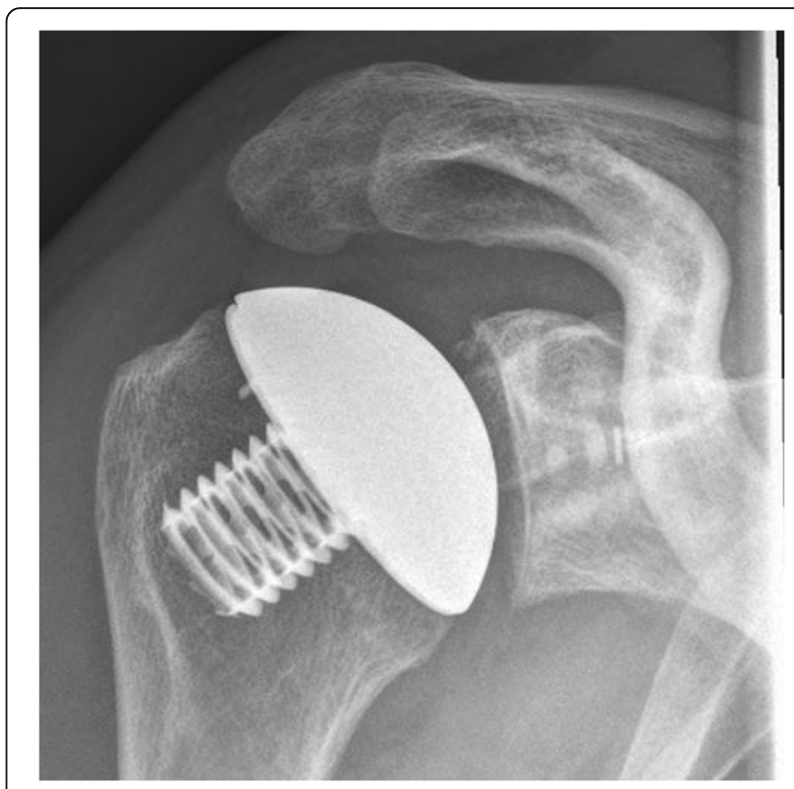

Fig. 3 Radiograph shoulder in true-AP: eclipse without calcar resorption

the physiotherapist. After 6 weeks active motion was added to the protocol. Strength exercises were carefully introduced after full range of motion was achieved.

\section{Data collection \& analysis}

Patients were examined by two parties and the functional scores were documented on datasheets. The radiographs were anonymously evaluated. Data were then

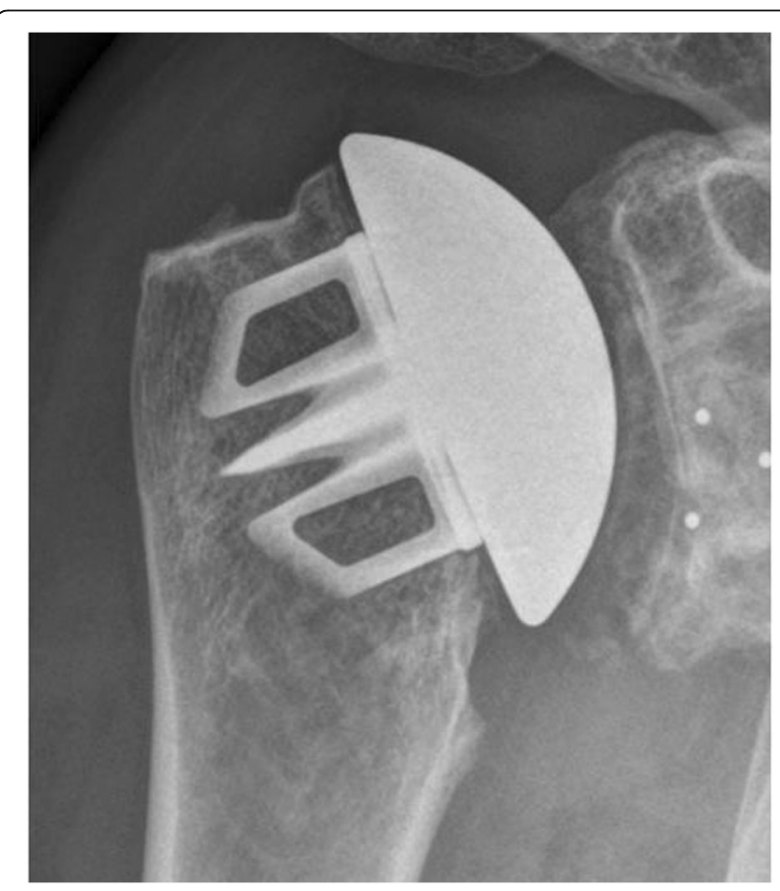

Fig. 4 Radiograph shoulder in true-AP: Sidus with calcar resorption 
statistically analyzed using Excel (Microsoft office 2016, Microsoft cooperation, Redmond, Washington, USA). We used a two sample t-test to determine significance. Because we presumed that both sets of data were independent and had the same potential for variance. The level of significance was set at below 0.05 .

\section{Results}

\section{Demographic results}

Mean age was 67 (range: 44-83 years median: 69) years at the time of operation. Average follow-up was 36.3 (24-72) months. Demographic data are summarized in Table 2. Of the 18 patients in group A, five were male and 13 were female. Mean age was 66.7 years (range: $44-81$ years median: 69). Average follow-up was 42.2 (24-72) months. Of the 21 patients in group B, six were male and 15 were female. Mean age was 67.4 years (range: $55-83$ years median: 71 ).

The average follow-up for group B was 36.3 (24-48) months.

\section{Functional results}

Patients in the group A revealed an increase in CS from 27.1 to 65.2 points $(p<0.001)$. The subcategories increased from 6.4 points to 13.4 points in pain, 8 points to 17.4 in activities of daily living, 11 points to 26.8 points in active range of motion and from 1.6 points to 7.2 points in strength $(p<0.001)$. The SSV increased from 27.3 to $76.7 \%(\mathrm{p}<0.001)$.

Preoperatively in group B there were 14 type A glenoids and 7 type $\mathrm{B}$ glenoids according to Walch et al. Patients in group B revealed an increase in CS from 29.0 to 72.6 points $(p<0.001)$. In the specific subcategories an increase from 6.2 points to 13.7 points in pain, 8.5 points to 19.2 points in activities of daily living, 13 points to 33.2 in active range of motion and from 0.93 points to 7.3 points in strength was observed $(\mathrm{p}<0.001)$. The average SSV here increased from 33.1 to $85 \%(p<0.001)$.

There was no significant difference in CS and SSV between the groups $(p=0.167)$. Functional results are summarized in Table 3 and Figs. 3 and 4.

\section{Radiological results}

Preoperatively in group A there were 13 type A glenoids and 5 type B glenoids according to Walch et al. [18].

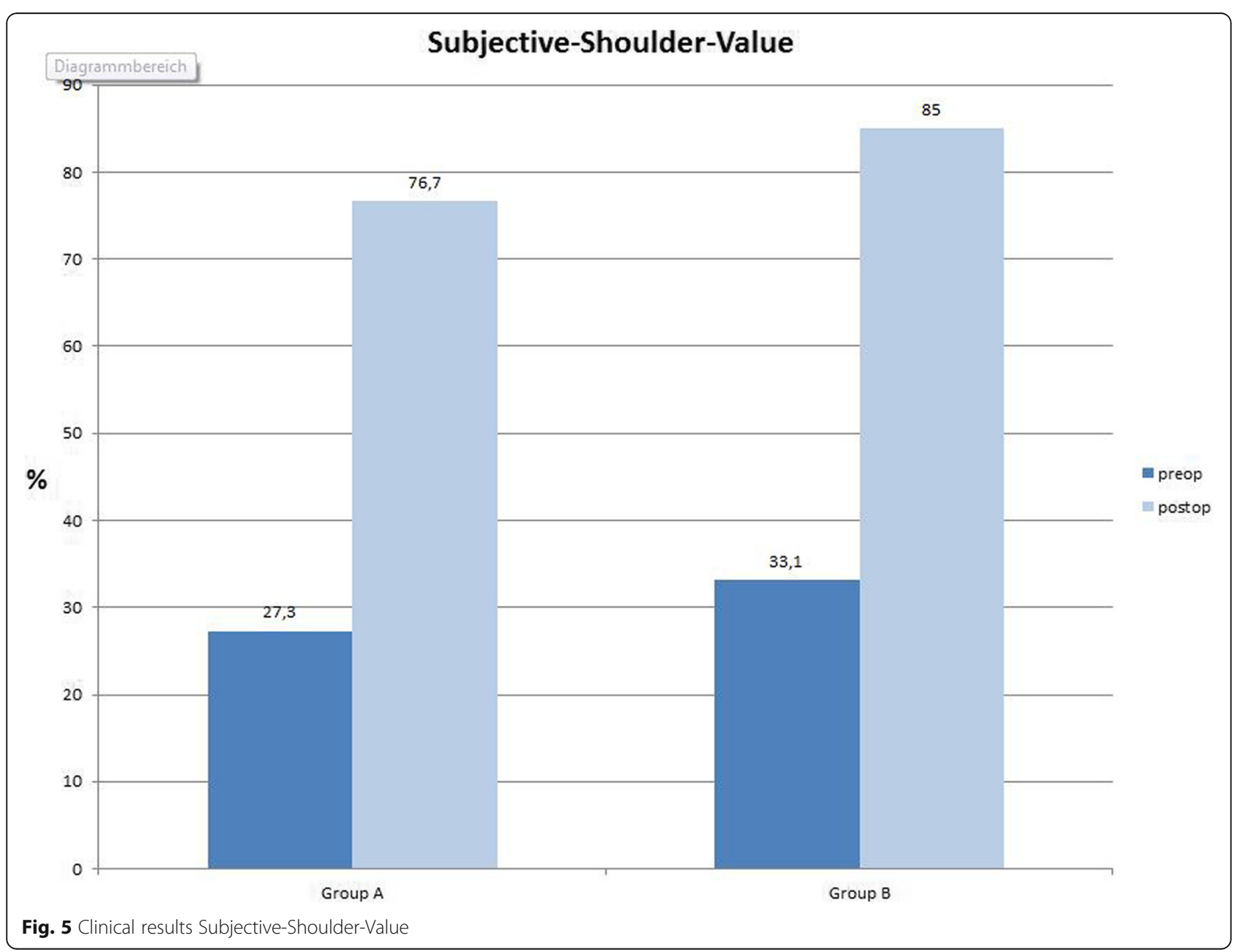




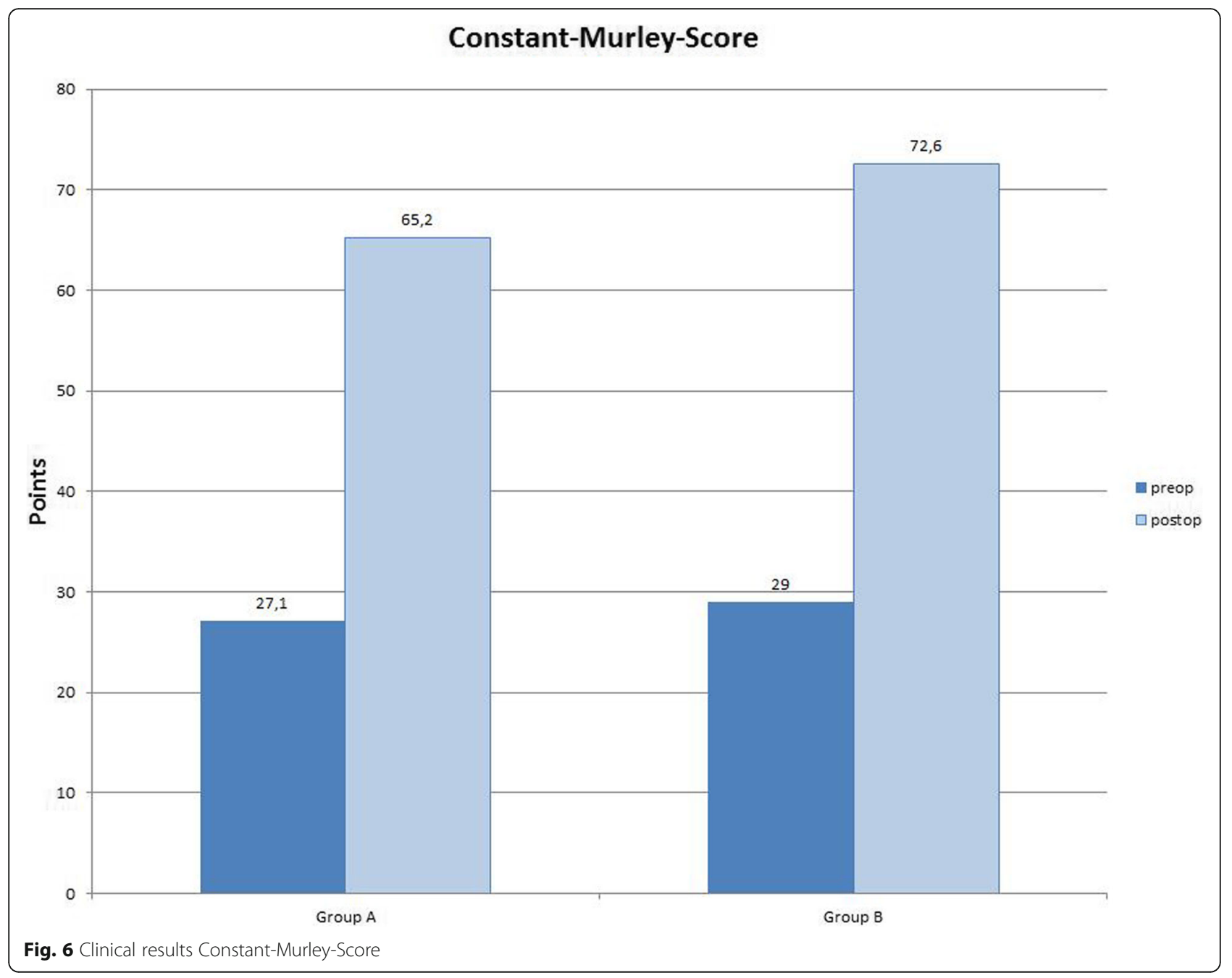

In group A we found radiolucent lines as signs of glenoid loosening in five patients (Mole-Score $=0.25$ ). There were neither signs of humeral loosening, osteolysis nor osteophytic exostosis (Fig. 5).

In group B four patients presented mild signs for glenoid loosening (Mole-Score $=0.89$ ). We found osteolysis of the medial calcar in seven patients (Fig. 6). Humeral loosening and osteophytic exostosis were not found.

There were no significant differences in loosening and osteophytic exostosis between the groups $(p=0,68)$. We found significantly more osteolysis in group B $(p<0.003)$. Radiographic results are summarized in Tables 4 and 5 .

\section{Complications}

We examined the electronical medical records and scanned for adverse events. We found complications in two patients. Complication rate was therefore $5.1 \%$. Revision rate in this cohort was $2.6 \%$. During anchor placement an intraoperative fracture at the greater tuberosity was recorded in group B. With no dislocation no further

Table 4 Preoperative and postoperative Constant-Murley-Score subcategory of both groups

\begin{tabular}{|c|c|c|c|c|c|}
\hline & Group A & & Group B & & \\
\hline & Preoperative & Last follow-up & Preoperative & Last follow-up & \\
\hline Pain & 6.4 points & 13.4 points & 6.2 points & 13.7 points & 0.147 \\
\hline Everyday activity & 8 points & 17.4 points & 8.5 points & 19.2 points & 0.499 \\
\hline Range of motion & 11 points & 26.8 points & 13 points & 33.2 points & 0.272 \\
\hline Strength & 1.6 points & 7.2 points & 0.93 points & 7.3 points & 0.144 \\
\hline
\end{tabular}


Table 5 Postoperative radiologic results of both groups

\begin{tabular}{llll}
\hline & Group A & Group B & $p$ value \\
\hline Loosening (humeral) & $N=0$ & $N=0$ & 0.500 \\
Loosening (glenoidal) & Mole score $=0.25$ & Mole score $=0.89$ & 0.269 \\
Osteophytic exostosis (humeral) & $N=0$ & $N=0$ & 0.500 \\
Calcar resorption & $N=0$ & $N=7$ & 0.005 \\
\hline
\end{tabular}

treatment was initiated and radiologically the fracture healed well. In group A a suspected low-grade infection with glenoid component loosening was treated with arthroscopic removal of the glenoid combined with the expiration of samples for microbiological examination. In a second step the endoprosthesis was revised to a reverse shoulder arthroplasty.

\section{Discussion}

By now several studies showing the benefits of stemless total shoulder arthroplasty have been published $[2,6,10$, 13, 17, 19]. Additionally, a finite element analysis concluded that stem length reduction resulted in a more physiological stress distribution on the humeral bone [20]. Results seem encouraging and are similar to other surgeon's experiences. Our null hypothesis (H0) turned out to be only partially true. Both designs evaluated in this study revealed a significant increase in function and patient satisfaction and decrease in pain levels. Improved clinical results were shown with both designs and radiologically both model's results are satisfying. But osteolysis of the medial calcar only appeared in the impaction design group. This might be the result of an uneven load distribution on the humeral bone. The Eclipse shoulder prosthesis might have a better distribution because of the screw fixation design in combination with the baseplate. The baseplate might distribute the load evenly leading to constant rim loading resulting in less bony resorption. The pressure in the Sidus Stem-free Shoulder System is conducted through the anchor and from there to bone.

Another explanation for this difference in radiological results is a biological reaction to a polyethylene wear of the glenoid component or impingement of the implant against the medial calcar (humeral notching). We used fully cemented keeled glenoids from each company with both designs. One explanation could be a different humeral-glenoid mismatch comparing both designs, however this remains a subject for further studies.

There is some evidence that radiological changes do not influence clinical results in short- and mid-term follow-up [21].

This study has some limitations. The number of patients in each group is relatively small and the study has a retrospective design. Neither the patients nor the examiners were blinded and the comparison was only based on short to mid-term results. We did not quantify bone quality as a preoperative mesurement. We used the thumbtest as described by Churchill et al. to evaluate all patients [11]. Only if the test was negative we implanted one of the two endoprothesis. After switching to an impaction method there might be a learning curve for the surgeon which can lead to difference in result. Furthermore, the method of examining radiolucent lines commonly used as indication for loosening seems to have limitations of its own $[21,22]$.

The strength of this study lies in the uniformity of patient treatment and examination. All patients were diagnosed and treated by the same surgeon and all data were collected by the same examiner who was not the operating surgeon. Even though this evidence is not conclusive it is promising and worthy of further observation in follow-up. A finite element analysis might also be useful to explain our results.

\section{Conclusion}

After a minimum of 24 months follow-up satisfying results can be achieved using either an impaction or a screw fixation stemless total shoulder arthroplasty. Clinical results in short-term follow-up do not differ from each other. The screw fixation seems to prevent osteolysis of the medial calcar.

\section{Abbreviations \\ vs: Versus; n: Number; CS: Constant-Murley-Score; SSV: Subjective-Shoulder- Value}

\section{Acknowledgements}

Not applicable.

\section{Authors' contributions}

MS and AA designed planed and organized the study. MS performed surgery on all patients. AA JPI AK performed clinical examination on patients. AA analysed and interpreted data. The manuscript was written by AA and improved by MS. The manuscript has been read and approved by all authors (AA, JPI, AK, MS).

Funding

No funding was required for the completion of this study.

Availability of data and materials

The datasets used and/or analysed during the current study are available from the corresponding author on reasonable request. 


\section{Compliance with ethical standards}

\section{Ethics approval and consent to participiate}

This study was approved by the ethics committee of our institution (EA2/ 154/18). Written informed consent was obtained from all patients enrolled in this study.

\section{Consent for publication}

Not applicable.

\section{Competing interests}

Mr. Arad Alikhah: This author, their immediate family, and any research foundation with which they are affiliated did not receive any financial payments or other benefits from any commercial entity related to the subject of this article.

Mr. Jan-Philipp Imiolczyk: This author, their immediate family, and any research foundation with which they are affiliated did not receive any financial payments or other benefits from any commercial entity related to the subject of this article.

Mrs. Anna Krukenberg: This author, their immediate family, and any research foundation with which they are affiliated did not receive any financial payments or other benefits from any commercial entity related to the subject of this article.

Prof. Markus Scheibel is a consultant for Arthrex Company.

Received: 16 June 2019 Accepted: 7 April 2020

Published online: 12 May 2020

\section{References}

1. Lo IK, Litchfield RB, Griffin S, Faber K, Patterson SD, Kirkley A. Quality-of-life outcome following hemiarthroplasty or total shoulder arthroplasty in patients with osteoarthritis. A prospective, randomized trial. J Bone Joint Surg Am. 2005:87(10):2178-85.

2. Chin PY, Sperling JW, Cofield RH, Schleck C. Complications of total shoulder arthroplasty: are they fewer or different? J Shoulder Elb Surg. 2006;15(1):19-22.

3. Farng $E$, Zingmond D, Krenek $L$, Soohoo NF. Factors predicting complication rates after primary shoulder arthroplasty. J Shoulder Elb Surg. 2011;20(4):557-63.

4. Singh JA, Sperling JW, Cofield RH. Revision surgery following total shoulder arthroplasty: analysis of 2588 shoulders over three decades (1976 to 2008). J Bone Joint Surg Br. 2011;93(11):1513-7.

5. Geurts GF, van Riet RP, Jansen N, Declercq G. Placement of the stemless humeral component in the Total Evolutive Shoulder System (TESS). Tech Hand Upper Extrem Surg. 2010;14(4):214-7.

6. Habermeyer $P$, Lichtenberg $S$, Tauber M, Magosch P. Midterm results of stemless shoulder arthroplasty: a prospective study. J Shoulder Elb Surg. 2015:24(9):1463-72.

7. Kadum B, Mafi N, Norberg S, Sayed-Noor AS. Results of the Total Evolutive Shoulder System (TESS): a single-centre study of 56 consecutive patients. Arch Orthop Trauma Surg. 2011;131(12):1623-9.

8. Churchill RS, Athwal GS. Stemless shoulder arthroplasty-current results and designs. Curr Rev Musculoskelet Med. 2016;9(1):10-6.

9. Hawi N, Tauber M, Messina MJ, Habermeyer P, Martetschlager F. Anatomic stemless shoulder arthroplasty and related outcomes: a systematic review. BMC Musculoskelet Disord. 2016;17(1):376

10. Berth A, Pap G. Stemless shoulder prosthesis versus conventional anatomic shoulder prosthesis in patients with osteoarthritis: a comparison of the functional outcome after a minimum of two years follow-up. J Orthop Traumatol. 2013;14(1):31-7.

11. Churchill RS, Chuinard C, Wiater JM, Friedman R, Freehill M, Jacobson S, et al. Clinical and radiographic outcomes of the Simpliciti canal-sparing shoulder arthroplasty system: a prospective two-year multicenter study. J Bone Joint Surg Am. 2016:98(7):552-60.

12. Razmjou H, Holtby R, Christakis M, Axelrod T, Richards R. Impact of prosthetic design on clinical and radiologic outcomes of total shoulder arthroplasty: a prospective study. J Shoulder Elb Surg. 2013;22(2):206-14.

13. Huguet D, DeClercq G, Rio B, Teissier J, Zipoli B, Group T. Results of a new stemless shoulder prosthesis: radiologic proof of maintained fixation and stability after a minimum of three years' follow-up. J Shoulder Elb Surg. 2010;19(6):847-52
14. Plachel F, Scheibel M. Humeral bone grafting in stemless shoulder arthroplasty. Obere Extrem. 2017:12(3):183-5.

15. Constant CR, Murley AH. A clinical method of functional assessment of the shoulder. Clin Orthop Relat Res. 1987:214:160-4.

16. Mole D, Riand N, Levigne C, Walch G. Cemented glenoid components: results in osteoarthritis and rheumatoid arthritis. In: Walch G, Boileau P, et al. , editors. Shoulder arthroplasty. Berlin: Springer; 1999.

17. Krukenberg A, McBirnie J, Bartsch S, Bohler N, Wiedemann E, Jost B, et al. Sidus stem-free shoulder system for primary osteoarthritis: short-term results of a multicenter study. J Shoulder Elb Surg. 2018;27(8):1483-90.

18. Walch G, Badet R, Boulahia A, Khoury A. Morphologic study of the glenoid in primary glenohumeral osteoarthritis. J Arthroplasty 1999;14:756-760. doi: 10.1016/S0883-5403(99)90232-2.

19. Hawi N, Magosch P, Tauber M, Lichtenberg S, Habermeyer P. Nine-year outcome after anatomic stemless shoulder prosthesis: clinical and radiologic results. J Shoulder Elb Surg. 2017;26(9):1609-15.

20. Razfar N, Reeves JM, Langohr DG, Willing R, Athwal GS, Johnson JA. Comparison of proximal humeral bone stresses between stemless, short stem, and standard stem length: a finite element analysis. J Shoulder Elb Surg. 2016;25(7):1076-83.

21. Heuberer PR, Brandl G, Pauzenberger L, Laky B, Kriegleder B, Anderl W. Radiological changes do not influence clinical mid-term outcome in stemless humeral head replacements with hollow screw fixation: a prospective radiological and clinical evaluation. BMC Musculoskelet Disord. 2018;19(1):28

22. Hudek R, Werner B, Abdelkawi AF, Schmitt R, Gohlke F. Radiolucency in stemless shoulder arthroplasty is associated with an imaging phenomenon. J Orthop Res. 2017:35(9):2040-50.

\section{Publisher's Note}

Springer Nature remains neutral with regard to jurisdictional claims in published maps and institutional affiliations.

\section{Ready to submit your research? Choose BMC and benefit from:}

- fast, convenient online submission

- thorough peer review by experienced researchers in your field

- rapid publication on acceptance

- support for research data, including large and complex data types

- gold Open Access which fosters wider collaboration and increased citations

- maximum visibility for your research: over $100 \mathrm{M}$ website views per year

At BMC, research is always in progress.

Learn more biomedcentral.com/submissions 